\title{
Improvement of eddy current testing method for sheet piles on harbor
}

\author{
Takuya Saito $^{1}$, Hitoshi MIKADA ${ }^{1}$, Junichi TAKEKAWA ${ }^{1}$ \\ ${ }^{1}$ Graduate school of Engineering, Kyoto University
}

\begin{abstract}
The applicability and the feasibility of eddy-current testing method for the detection of defects such as wall thinning, surface crack, pinholes, etc. of steel structure have been practically confirmed by field and laboratory experiments. Qualitative analysis of these defects has been empirically understood by the current practice of eddy current methods based on analog analysis. There has, however, been a growing demand to quantitatively evaluate the defects. We tackle this problem by use of time series of induced magnetic field caused by the defects. In the present study, we proposed a new digital analysis process to use the induced magnetic field waveform, and validated the effectiveness of the method using numerical simulations. First, we developed a high-speed electromagnetic simulator using a fictitious wave domain method to reproduce the conventional eddy current testing method for realistic sheet-piles seawater model. Then, we confirmed the features of the induced magnetic field waveforms by a few types of defects. Paying attention to the residuals of the induced magnetic field waveform, we developed a novel migration procedure for detecting accurate position of cracks or defects. Using our approach, we could obtain crack images without phase lag. For the evaluation of the crack position and wall thinning of the sheet piles, we also applied attribute analysis traditionally used in the field of seismic survey. Through the application of the digital signal processing using induced magnetic field waveform, we could successfully develop a processing scheme with a high degree of accuracy of the eddy current testing method.
\end{abstract}

\section{INTRODUCTION}

The eddy current testing method, which is one of the non-destructive inspection techniques, has been used for flaw detection test and material inspection. The applicability and the feasibility of eddy-current testing method for the detection of wall thinning and surface crack of steel structure have been practically confirmed by field and laboratory experiments. Qualitative analysis of cracks has been empirically understood by analog analysis for over three decades. There has, however, been a growing demand of quantitative evaluation on the position and/or depth of cracks. We tackle this problem by use of time series of induced magnetic field by the cracks.

In the present study, we proposed a new digital analysis process to use the induced magnetic field waveform, and validated the effectiveness of the method using numerical simulations. First, we developed a high-speed electromagnetic simulator using a fictitious wave domain method (Mittet (2010)) to reproduce the conventional eddy current testing method for realistic sheet-piles seawater model. The discretized equations are calculated by the finite difference time domain (FDTD) method.

Our approach is based on not only the residuals of the induced magnetic field waveform but also attribute analysis traditionally used in the field of seismic survey.

We, first, explain our method briefly. Next, we demonstrate the effectiveness of our method using some numerical experiments.

\section{THEORY \& METHOD}

For the simulation of the eddy current testing method, we used three-dimensional electromagnetic field analysis using the fictitious wave domain method (Mittet (2010)). For the development of the defect visualization method, we conducted the new migration process to obtained time series waveforms. We applied the attribute analysis used in the seismic survey to the migration result. Fig. 1 shows the calculation flow.

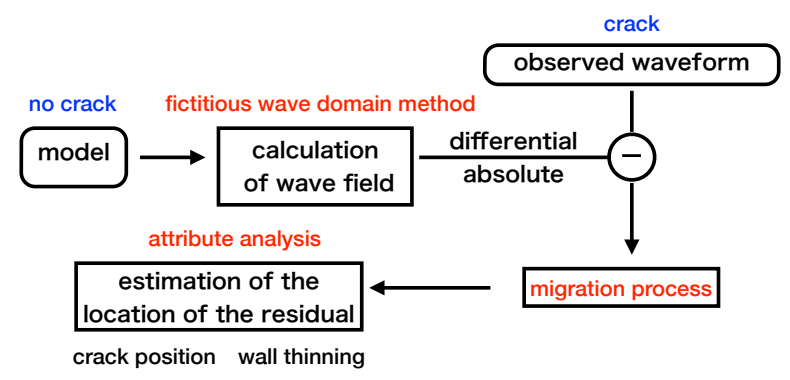


Figure 1 Simulation flow.

\section{(1) Fictitious wave domain method}

We calculated electromagnetic field of eddy current testing method in the fictitious wave domain method, described in detail in Mittet (2010). The Maxwell's equations in the diffusive time domain are

$$
\begin{aligned}
& -\nabla \times \mathbf{H}(x, t)+\sigma(x) \mathbf{E}(x, t)=-\mathbf{J}(x, t), \\
& \nabla \times \mathbf{E}(x, t)+\mu \partial_{t} \mathbf{H}(x, t)=-\mathbf{K}(x, t)
\end{aligned}
$$

where $\mathbf{E}$ and $\mathbf{H}$ are electric and magnetic fields, $\mathbf{J}$ and $\mathbf{K}$ denote electric and magnetic current densities of any external electromagnetic sources, $\sigma$ is electrical conductivity, and $\mu_{0}$ is magnetic permeability of free space. The main idea behind FDTD modeling is to transform eq. (1) mathematically to avoid the wide range of propagation velocities. After transforming these equations, this range of propagation is reduced. Following the derivation in Mittet (2010), we obtain the Maxwell's equation in the fictitious time domain as follows.

$$
\begin{aligned}
& -\nabla \times \mathbf{H}^{\prime}\left(x, t^{\prime}\right)+\varepsilon^{\prime}(x) \partial_{t^{\prime}} \mathbf{E}^{\prime}\left(x, t^{\prime}\right)=-\mathbf{J}^{\prime}\left(x, t^{\prime}\right) \\
& \nabla \times \mathbf{E}^{\prime}\left(x, t^{\prime}\right)+\mu \partial_{t^{\prime}} \mathbf{H}^{\prime}\left(x, t^{\prime}\right)=-\mathbf{K}^{\prime}\left(x, t^{\prime}\right)
\end{aligned}
$$

Where $\varepsilon^{\prime}$ satisfy $\varepsilon^{\prime}=\sigma / 2 \omega_{0}$ with $\omega_{0}$ is an arbitrary parameter. In this case, $\omega_{0}$ is set as $\omega_{0}=2 \pi$. $\mathbf{E}^{\prime}$ and $\mathbf{H}$ ' are electromagnetic fields in fictitious time domain. $\mathbf{J}$ ' and $\mathbf{K}^{\prime}$ denote electromagnetic current densities in the same domain. After calculating electromagnetic fields in the fictitious time domain, the physical electromagnetic fields $\mathbf{E}$ and $\mathbf{H}$ can be recovered from E', H', J' using the modified Fourier transform (Fig. 2). This fictitious wave domain method is used in the wave field analysis and the migration process. By using this method, analysis with realistic sheet pile model becomes possible.

For the simulation model, we assumed a steel plate contains a defect in sea water. The over view of the inspection is shown in Fig. 3. The obtained induced magnetic field waveform is differenced between two receiver coils. For absorbing boundary condition, the convolutional perfectly matched layer (Roden and Gedney, 2000) is used.

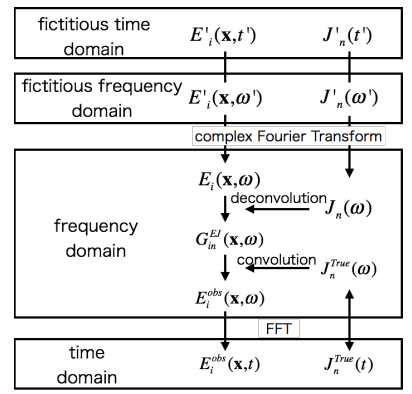

Figure 2 Flaw chart of fictitious wave domain method.

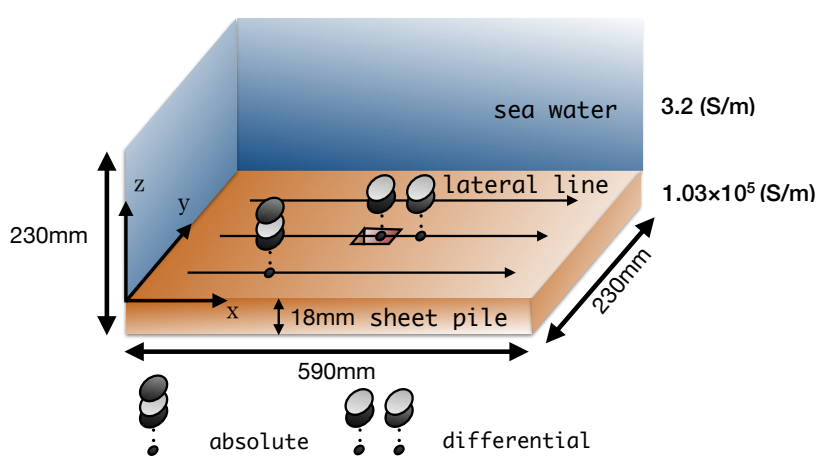

Figure 3 Overview of eddy current simulation. We call the inspection way using a probe with one transmitter coil and two receiver coils set vertically as absolute method. Similary, we call the inspection way using a probe with two sets of transmitter coil and receriver coil side by side as differential method.

\section{(2) Migration process}

We developed a new migration process. This process is applied to the obtained residual waveforms of magnetic field with and without crack. The migration process is shown as below.

1) Calculate the induced magnetic field waveforms with and without a crack by the fictitious wave domain method.

2) Calculate the residual of waveforms in 1).

3) Reverse the time of the residual in 2).

4) Propagate the reverse time residual of 3 ) at each receiver position.

5) Sum up the results in 4).

\section{(3) Attribute analysis}

We applied the attribute analysis to the migration results to clarify the position of the crack. We used instantaneous frequency analysis to elucidate the ambiguous boundary of the crack and sheet pile. We used cross-correlation to estimate the wall thinning variation. Eq. (3) is the definition of instantaneous frequency.

$$
\begin{aligned}
\omega(t) & =\frac{d \theta(t)}{d t} \\
& =\frac{d}{d t}\left\{\tan ^{-1}\left[f_{i}(t) / f_{r}(t)\right]\right\}
\end{aligned}
$$

\section{RESULTS \& DISCUSSION}

For the simulation model, we assumed two types of defects, i.e. pinhole type (Fig. 4) and wall thining type (Fig. 8). We calculated the migration 
of absolute and differential methods results (Fig. 5, Fig. 6). Although Energy is concentrated at the vicinity of the defect, specific position of the defect is still unclear. It is interesting to note that the migrated energy of the absolute method is concentrated on the crack while that of the differential method is concentrated near the crack.

To estimate the crack position and the thickness change of sheet piles more clearly, we use an attribute analysis after the migrated results. Fig. 7 shows the result of instantaneous frequency analysis of pinhole model using the differential method. The energey concentrated on the the crack position (red circle). Fig. 9 shows the result of instantaneous frequency analysis of wall thining model (absolute method). Red dotted lines represent the changing position of wall thickness. Large change in waveform could be found at red line. Fig. 10 shows the result of predicted depth of the wall thining by cross-correlation in the wall thinning model. We can estimate the position of the change in the depth of wall thining. In the migration result after the instantaneous frequency analysis, the position of the defect became clear. In addition, it was possible to represent thinning as a time delay using the cross-correlation process.

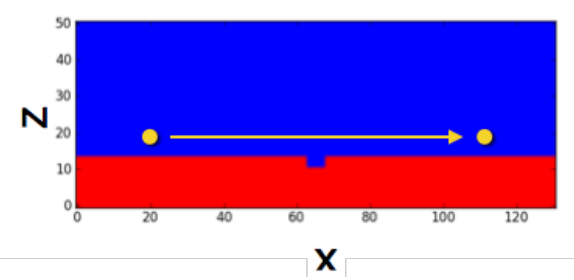

Figure 4 The pinhole model. Yellow point shows the probe position.
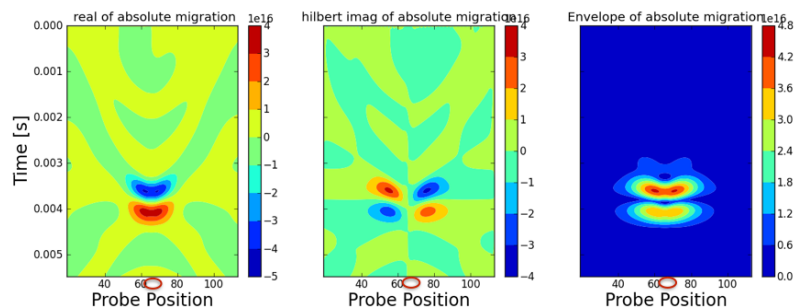

Figure 5 The migration result of absolute method of pinhole model. Real part (left), imaginary part (center), envelope (right).
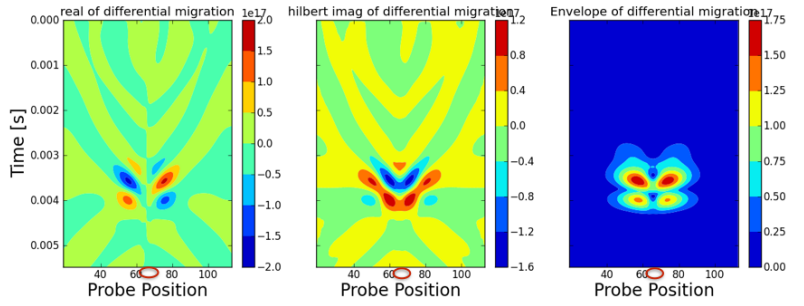

Figure 6 The migration result of differential method of pinhole model. Real part (left), imaginary part (center), envelope (right).

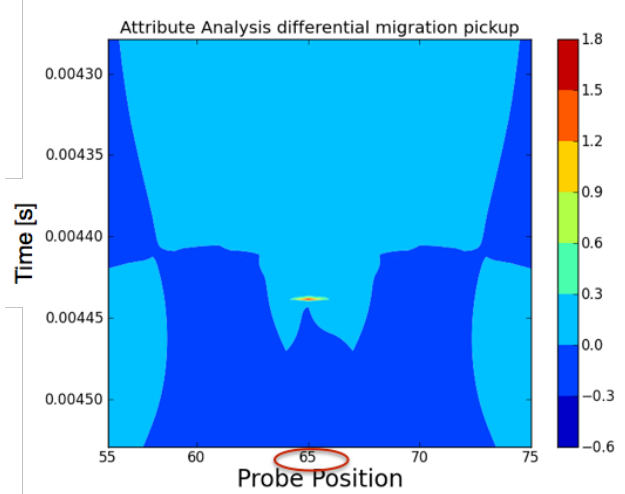

Figure 7 The result of the instantaneous frequency analysis of pinhole model (differential method). Red circle shows the position of the crack.

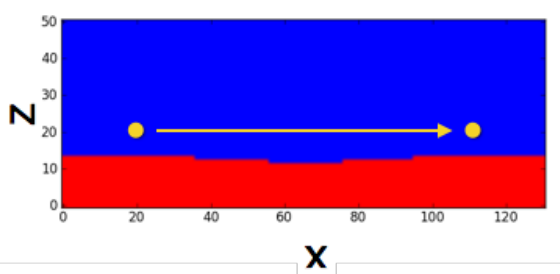

Figure 8 The wall thinning model. yellow point shows the probe position.

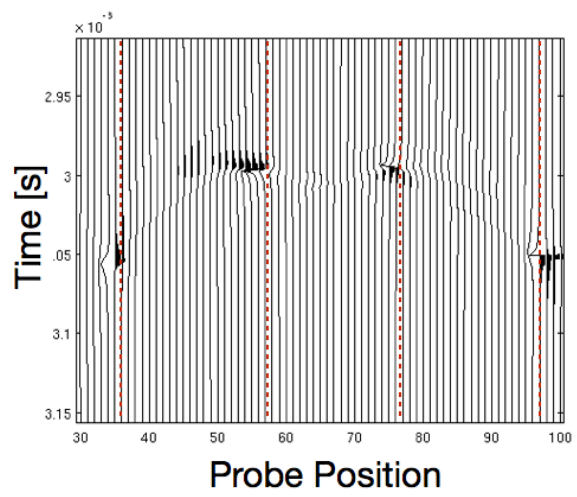

Figure 9 The result of the instantaneous frequency analysis of wall thinning model (absolute method). Red line represents the changing position of wall thickness.

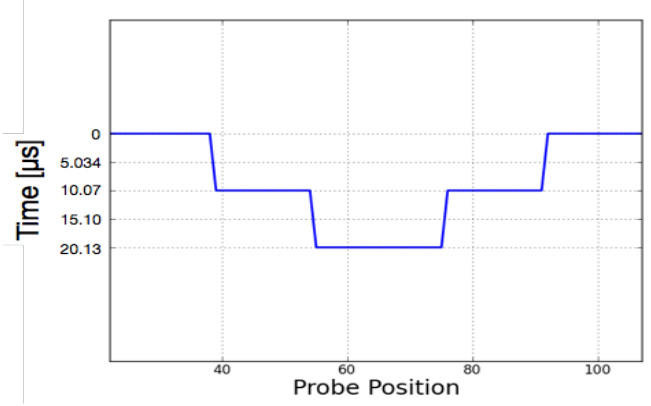

Figure 10 The result of cross correlation of the 
migration result of wall thinning model (absolute method).

\section{CONCLUSIONS}

We adopted a digital signal processing analysis for acquired magnetic waveforms induced in an eddy current testing method for achieving higher resolution. To validate the effectiveness of our approach, we conducted some numerical experiments. Eddy current testing method for sheet piles in harbor is simulated by the fictitious wave domain method. The method used a new migration procedure without phase lag and is led down to the attribute analysis to the migrated results. Using our method, we could precisely locate the crack position and estimate the wall thinning quantitatively with the accuracy higher than before.

\section{REFERENCES}

1) Mittet, R., 2010, High-order finite-difference simulations of marine CSEM surveys using a correspondence principle for wave and diffusion fields, Geophysics, 75, 33-50.

2) Roden, J. A., and Gedney, S. D., 2000, Convolutional PML (CPML): An efficient FDTD implementation of the CFS-PML for arbitrary media, Microwave and Optical Technology Letters, 27, 5, 334-338,

3) Taner, M.T., 1979, Complex seismic trace analysis, Geophysics, vol 44, p1041-1063. 